\title{
Philosophiques
}

\section{Grandeur et misère du socialisme scientifique}

\section{Maurice Lagueux}

Volume 10, numéro 2, octobre 1983

Le marxisme cent ans après Marx

URI : https://id.erudit.org/iderudit/203232ar

DOI : https://doi.org/10.7202/203232ar

Aller au sommaire du numéro

Éditeur(s)

Société de philosophie du Québec

ISSN

0316-2923 (imprimé)

1492-1391 (numérique)

Découvrir la revue

Citer cet article

Lagueux, M. (1983). Grandeur et misère du socialisme scientifique.

Philosophiques, 10(2), 315-340. https://doi.org/10.7202/203232ar d'utilisation que vous pouvez consulter en ligne.

https://apropos.erudit.org/fr/usagers/politique-dutilisation/ 


\section{GRANDEUR ET MISÈRE DU SOCIALISME SCIENTIFIQUE*}

par Maurice Lagueux

Il est vrai que le centenaire de la mort de Marx coïncide avec une période plutôt creuse pour le marxisme qui, dix ou quinze ans plus tôt, se trouvait au cœur même de la vie intellectuelle des principaux pays d'Occident, mais il n'en reste pas moins que le destin de ce courant de pensée fut tel qu'on aurait tort d'y voir le simple prolongement d'une contribution théorique du même type que toutes celles qui ont alimenté, de siècle en siècle, la culture occidentale. Il suffirait d'ailleurs, pour s'en convaincre, d'évoquer le fait qu'un mouvement socio-historique d'une ampleur sans précédent s'est emparé de la pensée de Marx pour en faire un étendard brandi triomphalement un peu partout sur la planète, quoiqu'il faudrait alors s'empresser d'ajouter que, par là même, risquait d'être profondément altérée la signification de l'entreprise théorique de l'un des penseurs les plus profonds du XIX ${ }^{\mathrm{e}}$ siècle. Quoi qu'il en soit, il s'agira moins ici de retrouver la pureté primitive de la contribution de ce penseur que d'identifier ce qui justement la destinait ainsi à inspirer à la fois une tradition intellectuelle obstinément vivace et un mouvement politique étonnamment contagieux.

Cette question semble avoir surtout préoccupé ceux qui y ont répondu en s'efforçant de justifier le fait qu'ils accordaient au marxisme une place à part dans l'histoire de la pensée. On connait, par exemple, l'hommage surprenant que Sartre, au début des années 60 , rendait à " l'indépassable philosophie de notre temps $»^{1}$ ou encore la façon tout aussi inattendue dont Merleau-

Une première ébauche de cet article a été présentée aux membres de la Société de Philosophie de Montréal, sous le titre "Marx est-il bien mort ? ", le mercredi 16 mars 1983.

1. Sartre, J.P., Critique de la raison dialectique, Paris : Gallimard, 1960, pp. 9, 17, 29, 32 et 109 . 
Ponty, au lendemain de la guerre, présentait le marxisme comme l'énoncé des conditions sans lesquelles le monde et l'histoire n'ont tout simplement plus de sens ${ }^{2}$. C'est toutefois Althusser et ses disciples qui, au cours des années récentes, auront le plus systématiquement réclamé un statut particulier pour cette "science marxiste» dont l'avènement correspondait, à leurs yeux, à rien de moins qu'à l'ouverture d'un nouveau " continent " théorique, le continent "Histoire ${ }^{3}$ sur lequel le marxisme se serait en quelque sorte assuré des droits exclusifs.

Ces perceptions du marxisme pourront paraître extravagantes ; elles n'en expriment pas moins le sentiment assez généralisé que l'œuvre de Marx a constitué dans l'histoire un "événement théorique " - pour reprendre une autre expression d'Althusser - dont la portée a certes été exagérée mais qui mérite bien d'être examiné pour lui-même.

D'ailleurs, dès avant la mort de Marx, cet événement théorique avait en quelque sorte fait l'objet d'une première analyse explicite de la part d'Engels dont le célèbre fascicule intitulé Socialisme utopique et socialisme scientifique visait précisément à mettre en relief la spécificité de la contribution de Marx. Celui-ci, grâce surtout à ses « deux grandes découvertes », la conception matérialiste de l'histoire et la théorie de la plus-value, aurait fait du socialisme une science, ce qui serait plus que suffisant pour garantir au marxisme le destin exceptionnel qui allait être le $\operatorname{sien}^{4}$.

Sans forcément adopter l'analyse engelsienne de la contribution de Marx, on peut trouver assez raisonnable de penser que c'est en effet pour avoir rendu enfin possible une sorte de jonction entre « science » et « socialisme » que Marx a pu être à l'origine des diverses traditions qui se sont réclamées de lui. Plus profondément, disons que ce que l'œuvre de Marx a apporté à ceux qui ont adhéré à sa pensée, c'est la conviction de pouvoir satis-

2. Voir, en particulier : Merleau-Ponty, M., Humanisme et terreur, Paris : Gallimard, 1947, p. 139.

3. Voir, par exemple : Althusser, L., Lénine et la philosophıe, Paris : François Maspero, 1972, pp. 21 et 53.

4. Voir : Engels, F., Socialisme utopique et socialisme sczentifique, Paris : Éditions sociales, 1971, pp. 88-89. Voir aussi le texte du bref discours d'Engels sur la tombe de Karl Marx. 
faire du même souffle ce qu'on peut sans doute considérer comme les deux plus nobles aspirations de tout être humain, soit son aspiration à la justice et son aspiration à la vérité. On s'était depuis longtemps résigné, en Occident, à séparer ces deux valeurs : au $\mathrm{XVI}^{\mathrm{e}}$ et au $\mathrm{XVII}^{\mathrm{e}}$ siècle, la recherche de la vérité était devenue l'affaire de savants dont les théories se faisaient superbement indifférentes au sort des laissés pour compte de l'humanité. Ceux-ci trouvaient, par contre, d'infatigables défenseurs chez des personnes dont on admirait vivement les qualités morales sans pour autant attendre d'elles de bien grandes contributions théoriques.

Or ce fut l'un des projets les plus constants du $\mathrm{XIX}^{\mathrm{e}}$ siècle que celui de fonder l'émancipation de " la classe la plus nombreuse et la plus pauvre ", comme disait Saint-Simon, sur l'application aux phénomènes sociaux de la méthode scientifique qui avait fait ses preuves en sciences naturelles. Pourtant, malgré les efforts les plus enthousiastes des socialistes les plus convaincus, cette idée ne parvenait manifestement pàs à s'imposer. Or, avec Marx, tout allait changer ; certes les thèses du Capital ne devaient-elles connaître qu'une diffusion assez lente du vivant de Marx, mais ceux qu'elles atteignaient se voyaient forcés de prendre au sérieux l'idée que le socialisme révolutionnaire pouvait être fondé sur une analyse scientifique. Un événement théorique considérable bouleversait littéralement la manière de penser des Occidentaux qui découvraient peu à peu que désormais la recherche de la vérité scientifique et le combat socialiste pour la justice pourraient bien ne faire plus qu'un. Mais examinons d'abord le contexte dans lequel un tel événement théorique a pu prendre sens avant de chercher à évaluer sa signification actuelle.

\section{UN ÉVÉNEMENT THÉORIQUE}

C'est peut-être une caractéristique de la philosophie classique que d'avoir cherché à fonder scientifiquement le discours moral tout en révélant progressivement les difficultés presque insurmontables d'une telle entreprise. Avant même que l'empirisme anglais ne souligne avec éloquence l'importance du fossé qui sépare les jugements de valeur des jugements de fait, le dualisme cartésien réduisait déjà les chances de fonder la morale 
sur la science, du seul fait qu'il logeait la première du côté du monde de l'esprit alors qu'il célébrait les triomphes que connaissait la seconde dans son analyse du monde matériel. Sans doute allait-on s'efforcer de développer quand même une science des choses de l'esprit, mais celle-ci allait au départ souffrir d'un double handicap. D'une part, elle portait sur un objet plutôt insaisissable qui offrait à d'éventuelles lois scientifiques beaucoup moins de prises que les choses matérielles. D'autre part, elle devait compter avec la conscience, la liberté et la finalité, toutes choses qui se conciliaient mal avec les mécanismes que la science classique se plaisait à démonter. C'est pourquoi, tout au long du XVIII ${ }^{e}$ siècle, les auteurs qui ont cherché le plus activement à rendre compte scientifiquement des mouvements de l'esprit se sont d'abord efforcés de dépasser ce dualisme en reliant de tels « mouvements » aux déplacements de la matière. D'où, chez eux, une sorte de préjugé matérialiste, mécaniste et déterministe qui commandait un discours fort éloigné, il va sans dire, des préoccupations (inspirées par une morale humaniste) de ceux qui s'intéressaient avant tout au sort des défavorisés de la société.

À l'aube du XIX ${ }^{\mathrm{e}}$ siècle, éthique sociale et pensée scientifique demeuraient donc plutôt étrangères l'une à l'autre. Ceux qui s'adonnaient à la science ne juraient que par les faits, leurs succès véritables concernaient le monde matériel et, puisque l'explication du système solaire, qui leur servait de modèle, pouvait désormais se passer d'horloger, ils avaient tendance à effacer de leur objet les traces toujours gênantes de la finalité. À l'inverse, les apôtres de la justice sociale défendaient une valeur morale, leur conviction reposait sur une conception de la dignité humaine irréductible à quelque dimension matérielle que ce soit et ils proposaient, somme toute, la poursuite consciente d'une finalité sociale.

Or, malgré cette situation, on allait bientôt voir s'affirmer avec force deux projets qui, chacun à sa façon, semblaient rapprocher ces deux pôles. D'une part, le mouvement socialiste, qui allait bientôt prendre de l'ampleur, était un projet de justice sociale que plusieurs de ses adeptes rattachaient d'emblée à une science nouvelle encore mal définie. D'autre part, l'économie politique commençait à être perçue comme une science fort respec- 
table même si elle s'intéressait directement à l'organisation des sociétés. Ces deux mouvements, dont j'évoquerai maintenant quelques traits essentiels, avaient d'ailleurs des racines profondes qui plongeaient dans la philosophie du XVIII ${ }^{\mathrm{e}}$ siècle.

Le socialisme saint-simonien, on le sait, descendait en droite ligne de la philosophie des lumières. Tout comme, pour Condorcet, les lumières devaient l'emporter sur la superstition et faire reculer ainsi les causes de l'exploitation de l'humanité, pour les socialistes proches de Saint-Simon, la science allait enfin permettre de construire une société plus juste aussi sûrement qu'elle permettait déjà de transformer le monde naturel. La volonté d'associer le destin d'un socialisme épris de justice au progrès de la science était donc manifeste, mais tout ce mouvement continuait néanmoins à se dérouler essentiellement au sein du monde des idées et des valeurs spirituelles. Les progrès des lumières et de la science libéraient d'abord les esprits et ce n'est qu'indirectement qu'on pouvait en attendre une transformation des structures sociales. Le socialisme saint-simonien se présentait même comme un " nouveau christianisme " et il s'en remettait en définitive à l'éducation de l'humanité pour assurer la réalisation de finalités sociales tout au plus un peu mieux définies ${ }^{5}$. Sans doute, Saint-Simon estimait-il pouvoir ramener la gestion future des affaires sociales à une simple « administration des choses », mais l'essentiel pour lui était que cette gestion soit confiée à des savants qui sauraient, mieux que d'ignares politiques, réaliser les fins poursuivies. Bref, ce socialisme se voulait certes scientifique, mais faute de pouvoir expliquer les phénomènes sociaux de la façon dont la physique expliquait les phénomènes du monde matériel, il se contentait d'inciter avec éloquence à la poursuite de finalités sociales présumées parentes des résultats obtenus ailleurs grâce au patient labeur des hommes de science.

L'économie politique qui, avec Ricardo, s'imposait désormais comme une science respectable avait, elle aussi, trouvé sa voie dès le XVIII ${ }^{\mathrm{e}}$ siècle. Elle n'y était parvenue cependant qu'à la suite d'une rupture avec les valeurs de la morale traditionnelle

5. Voir en particulier : Saint-Simon, H. de, Le nouveau cbristianisme, Paris : Éd. du Seuil, collection Politique, no. 30. Voir aussi la remarquable introduction de cet ouvrage par Henri Desroches. 
auxquelles était jusqu'alors associée la réflexion sur l'homme et la société. On peut, en effet, avec Louis Dumont, voir dans la Fable des Abeilles de Bernard de Mandeville l'expression privilégiée d'une sorte d'émancipation à l'égard de la morale d'un nouveau type de discours économique 6 . L'idée de Mandeville était que la prospérité matérielle, dont l'économie politique entendait rendre compte, ne dépendait en rien des bonnes intentions de chacun ni des fins consciemment poursuivies, mais résultait au contraire de la satisfaction des pulsions égoïstes d'individus motivés par leurs seuls intérêts matériels. Rien alors n'empêchait plus une analyse débarrassée de toute considération de finalité de rendre compte scientifiquement de l'organisation de la vie sociale à partir de ces dimensions plutôt matérielles. Adam Smith, en tout cas, allait tirer parti de cette façon de voir au moment de fonder sa théorie économique sur la très prosaique constatation que « ce n'est pas de la bienveillance du boucher, du marchand de bière ou du boulanger, que nous attendons notre dîner, mais bien du soin qu'ils apportent à leurs intérêts » ${ }^{7}$. L'économie politique pouvait ainsi se présenter comme une science assez comparable aux sciences physiques et se démarquer de tout discours moral, mais le cynisme même sur lequel elle fondait cette prétention en faisait la base d'une idéologie farouchement hostile aux objectifs de justice sociale que prônait le socialisme ${ }^{8}$.

Bref, pour ceux qui, comme Marx et Engels, commençaient, vers l'an 1844, à s'interroger sur le destin de la classe ouvrière et du système capitaliste dont cette dernière était victime, l'horizon théorique était dominé par un mouvement socialiste incapable de s'affirmer sérieusement comme science et par une science économique pour le moins indifférente aux problèmes d'équité que soulevait le capitalisme.

6. Voir : Dumont, L, Homo aequalis, Paris : Gallimard, 1977, ch. 5. On trouvera une traduction française de "The Fable of the Bees" par L. et P. Carrive in Mandeville, La fable des abeilles, Paris : Vrin, 1974 ; pour la totalité des commentaires ajoutés par Mandeville, il faut se référer à l'édition anglaise en 2 volumes de Kaye.

7. Smith, A., Recherches sur la nature et les causes de la richesse des nations, Paris : Gallimard, 1976, (trad. Mairet), p. 48.

8. Pour plus de précisions sur les rapports de l'économie politique et de l'idéologie, voir mon texte intitulé "Ces idéologies qui faisaient parler les choses " in Crise et leadership, Montréal : Boréal express, 1983, pp. 293-309. 
Or, entre ces deux univers intellectuels opposés, Marx n'allait pas tarder à devoir choisir, ou presque. Dès 1846 , ce choix était fait et allait l'amener à rompre avec la plupart des socialistes insensibles aux exigences véritables de la méthode scientifique. C'est ainsi que, lors d'une discussion assez vive, il fit brutalement savoir au communiste Weitling que celui-ci avait tort de se méfier systématiquement des théoriciens : "L'ignorance, lui lança-t-il, n'a jamais servi personne ! " ${ }^{9}$ Plus cruellement encore, il devait bientôt faire de Proudhon la victime privilégiée de ses sarcasmes et même prendre littéralement parti pour Ricardo contre ce dernier :

La théorie des valeurs de Ricardo est l'interprétation scientifique de la vie économique actuelle; la théorie des valeurs de $\mathbf{M}$. Proudhon est l'interprétation utopique de la théorie de Ricardo ${ }^{10}$.

Pourtant, on s'en doute bien, si Marx décidait ainsi de prendre coûte que coûte parti pour la science, il n'entendait pas pour autant endosser les valeurs associées à la pensée de Ricardo et il ne renonçait en rien au socialisme auquel il avait résolu, au contraire, de donner des bases scientifiques. La confiance qu'il manifestait alors sur ce plan reposait largement sur le fait que, contrairement à Proudhon et aux socialistes utopistes, il était à même de trouver chez Hegel l'instrument dialectique qui allait lui permettre de réconcilier socialisme et scientificité.

Pourtant Hegel n'avait rien d'un socialiste et, des deux traits (privilège explicatif accordé au monde matériel et élimination de la finalité au niveau des individus) qui ont permis cidessus de reconnaître à l'économie politique une scientificité comparable à celle des sciences physiques, un seul était compatible avec la philosophie hégélienne ! Cette philosophie, en effet, était bien loin d'accorder un privilège au monde matériel même si, mieux encore que la philosophie qui sous-tendait l'économie politique, elle savait se passer, dans ses explications, des intentions conscientes des agents historiques dont les actions égoïstes voyaient leurs résultats récupérés par une " ruse de la raison".

9. Cité par Henri Lefebvre in Pour connatitre la pensée de Karl Marx, Paris : Bordas, 1956, p. 146 .

10. Marx, K., Mzsère de la Pbilosophie in Marx, K., Oeuvres, Paris : Gallimard (Bibliothèque de la Pléiade), tome I, 1963, p. 25. Les tomes I (1963), II (1968) et III (1982) de cette édition seront désormais désignés respectivement par Pléiade I, Pléiade II et Plétade III. 
Il importait donc de " renverser " — c'est-à-dire de fonder dans le monde matériel, ou, si l'on préfere, de "remettre sur ses pieds " - la dialectique hégélienne pour revalider ses titres à la scientificité et c'est ce que Marx s'est empressé de faire ${ }^{11}$. Hegel méritait bien, aux yeux de Marx, de voir sa pensée ainsi revitalisée, car celle-ci apportait à l'architecte d'une nouvelle science sociale cette intelligence de l'histoire qui faisait cruellement défaut tant aux économistes qu'aux socialistes de son temps. Or il fallait rien de moins qu'une nouvelle théorie de l'histoire pour réconcilier science économique et projet socialiste ou, plus précisément, pour concéder à Ricardo "l'interprétation scientifique de la vie économique actuelle " tout en se réservant la possibilité de promettre l'avenir au socialisme.

Une telle réconciliation peut paraître assez simple, mais il fallait tout le génie de Marx pour la réaliser pleinement. Plus exactement, étaient requises ces " deux grandes découvertes» qui furent les siennes, selon Engels. La « conception matérialiste de l'histoire " d'abord, qui résultait bien, d'une certaine façon, d'une inversion de la théorie hégélienne, mais à condition, bien sûr, que soient mis au point des concepts neufs ou renouvelés comme ceux de «base matérielle » et de « superstructure », de "forces productives " et de « rapports de production "; à condition que soit repensé le mécanisme qui allait jouer le rôle de la ruse de la raison et amener par exemple les capitalistes désireux d'accroître leurs capitaux à se faire par là même leurs " propres fossoyeurs " ${ }^{12}$; à condition surtout que soit élaboré le concept de « mode de production » et précisés, par opposition aux modes de production antérieurs, les caractères propres au mode de production capitaliste dont le déclin historique devait rendre possible la construction du socialisme.

Ces dernières précisions, c'est dans le Capital que Marx devait les apporter en même temps qu'il y exposait sa seconde " grande découverte », la " théorie de la plus-value », qui achevait, à ses yeux, de sceller, entre science et socialisme, le mariage que la conception matérialiste de l'histoire avait rendu possible.

11. Voir la Postface de la seconde édition allemande du Capital, par exemple in Pléiade I, pp. 558 et 559 .

12. Marx, K. et Engels, F., Manifeste communiste in Pléiade I, p. 173. 
En somme, si cette dernière théorie fondait scientifiquement la dimension annonciatrice du projet socialiste, c'est-à-dire permettait d'annoncer le déclin du capitalisme et l'avènement d'une société meilleure, la théorie de la plus-value renforçait le verdict porté sur le capitalisme et apportait une profondeur scientifique nouvelle à la dimension dénonciatrice du projet socialiste. Grâce à cette analyse du mécanisme de production de la plus-value qui est censé découler du fonctionnement normal du capitalisme, Marx parvient en effet à rendre superflues les traditionnelles dénonciations de l'exploitation qui constituaient la pièce centrale de l'éthique socialiste. Le Capital qui se veut une œuvre scientifique se garde bien de dénoncer directement ; Marx y met néanmoins tout en place pour provoquer chez le lecteur un incompressible sentiment de révolte à l'égard du système capitaliste dans son ensemble, et c'est bien ce qui a fait de cet ouvrage un instrument révolutionnaire d'autant plus efficace qu'il est perçu à bon droit comme un travail scientifique ${ }^{13}$.

Bref, la contribution décisive de Marx aura été de donner une haute crédibilité à l'idée que, tant dans son aspect annonciateur d'une société meilleure que dans son aspect dénonciateur de la société actuelle ${ }^{14}$, le socialisme pouvait être perçu comme le condensé des conclusions d'une théorie scientifique. Plus précisément, il aura rendu cette idée crédible en conférant à cette théorie scientifique les traits qui étaient censés caractériser les théories en sciences naturelles (privilège du matériel, absence de finalité) tout en la fondant sur une perception originale de l'histoire qui la mettait au-dessus du mimétisme insignifiant à l'endroit des sciences naturelles auquel les sciences sociales sont si souvent réduites.

Par là même, Marx gagnait de vitesse les théoriciens bourgeois qui hésitaient encore à expliquer les phénomènes sociaux par leurs conditions matérielles; de ce fait, l'idée même de « science sociale » allait être associée — et pour longtemps -

13. C'est cet aspect des choses que j'ai développé dans une communication présentée au XIX congrès des Sociétés de Philosophie de Langue Française, tenu à Bruxelles et Louvain-laneuve en septembre 1982, sous le titre « Dimension éthique du débat sur la valeur (et de l'approche marxiste en général) ". L'essentiel de cette communication doit paraître dans les Actes du dit congrès.

14. Voir à ce sujet le chapitre III, "Marxisme annonciateur et marxisme dénonciateur " de Lagueux, M., Le marxisme des années soixante, Ville LaSalie : Hurtubise HMH, 1982. 
à l'idée subversive de critique sociale. En tout cas, avec cette contribution de Marx, une voie royale s'ouvrait enfin pour ceux que leur sens aigu de la justice sociale avait transformés en militants socialistes alors même que, mus par une passion sincère pour la vérité, ils entendaient bien s'adonner sans compromis aux recherches scientifiques les plus rigoureuses.

Voilà donc l'événement théorique qui a fait de la pensée de Marx bien autre chose qu'une philosophie parmi d'autres : pour la première fois sans doute dans l'histoire de l'Occident, on pouvait en toute bonne foi et $d u$ même souffle répondre adéquatement à l'appel de la justice et à celui de la vérité. Avant de voir comment on a pu alimenter cette conviction au cours des cent années de l'histoire du marxisme, voyons un peu ce qu'il en est, en particulier, de la cohérence de cette solution.

\section{COHÉRENCE ET INSUFFISANCE DE LA SOLUTION MARXIENNE}

À ceux qu'avait enthousiasmés cette perspective grandiose, l'histoire tourmentée du marxisme devait réserver bien des déceptions, tant sur le plan de la théorie que sur celui de la pratique. En réaction, la tentation a été grande, surtout ces dernières années, de dénoncer, sur tous les plans, la pensée de Marx présentée alors comme le lieu des contradictions les plus insurmontables. Aussi, avant de souligner les difficultés effectivement rencontrées par cette pensée, il convient d'examiner sommairement les principaux griefs de contradiction qui furent portés contre elle.

\section{A) Une théorie cohérente}

1) Une première " contradiction " qui fut à l'origine de bien des incompréhensions est celle, sous-jacente dans le débat avec Proudhon, qui se profile entre le matérialisme sans âme que semble adopter Marx et l'bumanisme généreux qui paraît indissociable de tout projet socialiste. Pourtant cette " contradiction " ne devrait plus nous inquiéter tellement et je ne m'y attarderai guère. Pour Marx, on l'a vu, l'orientation « matérialiste » n'est qu'un autre nom pour une orientation résolument "scientifique ». Pour un homme du XIX ${ }^{\mathrm{e}}$ siècle, la science s'oppose à la spéculation en ceci qu'elle explique des phénomènes 
réels à partir de causes réelles. Comme le monde matériel avait alors tendance à être perçu, à tort ou à raison, comme le seul qui soit indiscutablement réel, les intérêts matériels (comme l'appât du gain) paraissaient, par extension, plus réels et donc plus susceptibles de fonder une explication scientifique valable que les préceptes humanistes les plus recommandables.

De plus, rien ne contribuait autant, aux yeux de Marx, à masquer l'exploitation dont les travailleurs étaient victimes que les mystifications idéologiques que rendaient possibles les spéculations un peu gratuites de bien des philosophes idéalistes. Celui-ci s'est donc employé à dénoncer sévèrement cette spéculation en recourant à une analyse démystifiante qui permettait de retrouver, sous les justifications idéologiques, les mécanismes et les mobiles inconscients qui expliquent souvent les comportements et les options théoriques. C'en était assez pour l'amener à proclamer avec Engels dans l'Idéologie allemande son attachement à une science strictement "empirique ", aux dépens de la spéculation philosophique qui se trouve alors vertement dénoncée $^{15}$. Ceci ne l'a pas empêché, il va presque sans dire, d'élaborer sa propre conception philosophique de l'homme et de l'histoire, puisque cette conception sous-tendait son analyse « scientifique'» ${ }^{16}$. Aussi serait-il pour le moins trompeur d'invoquer le rejet par Marx de toute philosophie idéaliste pour en faire un "scientifique " matérialiste étranger aux objectifs et aux valeurs de la réflexion philosophique. De même, ce rejet de la philosophie ne l'a pas empêché, de manifester, au cœur même du Capital, son œuvre la plus "matérialiste ", une très haute conscience de cette dignité humaine à laquelle renvoie, pour plusieurs, la notion ambiguë d' « humanisme ».

Bref, il n'y a pas chez Marx de contradiction entre matérialisme et humanisme et il n'y a pas davantage de rupture entre l'œuvre philosophique explicite de la jeunesse et le travail scientifique de la maturité, puisque l'orientation « matérialiste » du second visait manifestement à rendre plus efficace la dénoncia-

15. Voir par exemple Marx, K. et Engels, F., L'Idéologie allemande in Pléiade III, pp. 1054 à 1058 .

16. Voir à ce sujet : Lagueux, M., "La philosophie du svieux, Marx " in Carrefour, revue de la société de philosophie de l'Outaouais, vol. 5, no 1, mai 1983, pp. 74-86. 
tion « humaniste» de l'aliénation à laquelle se consacrait la première.

2) Une autre contradiction qu'on n'a pas tardé à reprocher à Marx concerne plus immédiatement l'action révolutionnaire à laquelle son œuvre est traditionnellement associée. Étant donné son attitude résolument scientifique, Marx lui-même est entré assez tôt en conflit avec des révolutionnaires plus inconditionnels que n'impressionnaient guère ses analyses minutieuses du contexte socio-historique de l'action politique. Aussi peut-on se demander si les scrupules du penseur n'ont pas étouffé l'attitude révolutionnaire chez celui qui n'hésita pas à prendre sur lui de dissoudre la Ligue des Communistes et, plus tard, à recommander la mise en veilleuse de la première Internationale ${ }^{17} . \grave{A}$ un niveau plus strictement théorique, une " contradiction » apparentée à celle-là a souvent été signalée entre une théorie « déterministe » de l'histoire, selon laquelle la chute du capitalisme et le passage au socialisme relèveraient d'abord d'une maturité historique qui doit venir à son heure, et une pratique politique qui insisterait sur la nécessité de l'union des prolétaires pour l'action révolutionnaire capable seule de violenter et de réorienter l'histoire.

Pourtant, même si divers passages de l'œuvre si considérable de Marx peuvent être interprétés dans un sers ou dans l'autre, on aurait bien tort de voir là des contradictions plus significatives que celles qui accompagnent infailliblement l'expression, reprise sur plusieurs décennies et dispersée dans les contextes les plus variés, d'une thèse passablement nuancée. Marx a certes proposé une théorie globale de l'histoire et a même parlé de la "loi naturelle qui préside " au mouvement d'une société ${ }^{18}$, mais c'était avant tout pour rappeler que, dans l'évolution des sociétés comme dans celle de la nature, n'importe quoi n'est pas compatible avec n'importe quoi :

personne n'ignore, rappelait-il d'ailleurs plaisamment, que déjà

Don Quichotte a eu à se repentir pour avoir cru que la chevalerie errante était compatible avec toutes les formes économiques de la société ${ }^{19}$.

17. Voir à ce sujet : Rubel, M., "Chronologie ", Pléiade I, pp. LXXIII et CLV ; voir aussi Lefebvre, op. cit., deuxième partie, ch. II et III et troisième partie, ch. IV.

18. Marx, K., Préface de la première édition du Capital in Pléiade I, p. 550 ; le souligné est de Marx.

19. Marx, K., Le Capttal, Pléiade I, p. 617, note a de la page précédente. 
Ce principe une fois posé, Marx s'est employé à montrer que, dans l'histoire, les contraintes imposées par l'état du développement économique pèsent de tout leur poids sur les relations socio-politiques et culturelles qui se tissent successivement au cours des âges. Il a ensuite examiné, avec plus de détails, le développement historique du capitalisme, mais il s'est défendu vivement contre ceux qui ont commis l'erreur de " métamorphoser » son « esquisse de la genèse du capitalisme dans l'Europe occidentale en une théorie historico-philosophique de la marche générale, fatalement imposée à tous les peuples, quelles que soient les circonstances historiques où ils se trouvent placés $\left[\right.$. . . ] $\gg^{20}$.

De même qu'il envisageait ici la possibilité d'un déroulement de l'histoire qui, moyennant certaines conditions, parvienne à se passer des dures leçons du capitalisme, Marx s'interrogeait ailleurs sur la nécessité d'une révolution violente pour les pays les plus démocratisés qui pourraient bien l'éviter sauf, bien entendu, si la violence est provoquée par « ceux qui sont intéressés à l'ancien état de choses ${ }^{21}$. En somme, si, pour Marx, il y a bien, dans l'Occident contemporain, une sorte de tendance historique selon laquelle «la production capitaliste engendre elle-même sa propre négation avec la fatalité qui préside aux métamorphoses de la nature ${ }^{22}$, le processus qui doit présider à la destruction du mode de production capitaliste n'a rien de prédéterminé.

Ce qui paraît seul s'imposer, à ses yeux, c'est que l'évolution de ce mode de production ne peut que contribuer, par le biais même de l'accumulation du capital, à la mise en place de conditions matérielles susceptibles de favoriser son renversement et, de ce fait, l'avènement d'une société socialiste. Rien là qui rendrait superflue l'action révolutionnaire. Bien au contraire, une fois présentes les conditions qui la rendent possible et efficace, cette action révolutionnaire peut devenir le moment décisif du processus. Ce que Marx excluait, c'est que la révolution soit possible dans n'importe quelle condition : l'éveil d'une

20. Marx, K., "Réponse à Mikhailovski (nov. 1877)" in Plétade II, p. 1555.

21. Tiré d'un écrit de 1878 sur la loi anti-socialiste, cité par Rubel dans sa chronologie in Pléiade I, p. CL.XV.

22. Marx, K., Le Capital, Pléiade I, p. 1239. 
véritable conscience révolutionnaire exigeait selon lui des conditions socio-économiques appropriées. C'est d'ailleurs pourquoi, dans l'attente optimiste mais réaliste de la manifestation de telles conditions, il estimait vaines, prématurées et même nuisibles à la cause prolétarienne les tentatives révolutionnaires dispersées auxquelles s'adonnaient bien des activistes outrés par son attentisme de théoricien.

Entre une théorie de l'histoire qu'il faut savoir appliquer et une activité révolutionnaire qui y joue un rôle décisif mais conditionné, il n'y a pas de véritable contradiction, même s'il y a place - mais qui s'en formaliserait ? - pour les interprétations les plus divergentes.

3) Une autre question qui a généré beaucoup de débats passionnés dans l'histoire du marxisme est évidemment celle de l'efficace respective de l'économie et de ce qu'on a pris l'habitude d'appeler les autres instances. La priorité apparemment reconnue aux facteurs économiques s'est vue souvent confrontée à la relative autonomie qu'il est difficile de refuser aux autres instances. En fait, la mise en évidence par Marx du rôle important des facteurs économiques semble avoir tellement impressionné ses premiers disciples, qui y voyaient sans doute la marque de la supériorité de leur doctrine sur celle des « idéalistes » bourgeois, qu'ils ont souvent été amenés à en exagérer l'importance. C'est du moins ce qu'Engels lui-même leur reprochait dans sa célèbre lettre à Bloch, en ajoutant aussitôt que Marx et lui n'avaient malheureusement pas toujours trouvé «le temps, le lieu, ni l'occasion de donner leur place aux autres facteurs qui participent à l'action réciproque ${ }^{23}$.

On comprend aisément que, dans le chœur fortement idéaliste des théories sociales au XIX $\mathrm{X}^{\mathrm{e}}$ siècle, Marx ait voulu insister sur l'importance de la dimension économique et qu'il ait jugé utile de proposer un modèle théorique dont la "base " était constituée de relations économiques. Le choix de cette position pour les relations économiques n'avait pourtant rien de très métaphysique ; il tenait plutôt au fait que, comme le rappellent Marx et Engels, « les hommes doivent être en mesure de vivre

23. Engels, F., Lettre à Joseph Bloch du 21 sept. 1890 in Marx, K. et Engels, F., Études Philosophiques, Paris : Éditions sociales, 1974, p. 240. 
pour être capable de (faire l'histoire» » et que «pour vivre, il faut avant tout manger et boire, se loger, se vêtir et maintes choses encore ${ }^{24}$. Ce constat plutôt banal n'était certes pas suffisant pour exclure l'idée d'une efficace des structures politiques et idéologiques dont Marx voulait seulement rappeler qu'elles sont rendues possibles par un contexte économique favorable que d'ailleurs - et pourquoi pas ? - elles contribuent à modeler.

Il est vrai qu'on trouve parfois chez Marx des passages qui semblent suggérer une relation plus unilatérale comme celui, souvent évoqué, qui assure que « le moulin à bras vous donnera la société avec le suzerain ; le moulin à vapeur, la société avec le capitaliste industriel ${ }^{25}$. Mais, ici encore, c'est à tort que l'on aura isolé cette formule lapidaire pour en faire l'expression d'une métaphysique dogmatique, sans même la situer dans son contexte qui, incidemment, rappelle que les rapports sociaux, tout comme les marchandises, sont "produits par les hommes ", en tant certes qu'ils sont les détenteurs de forces productives, mais de forces productives dont le développement est rendu possible (comme le rappelle d'autres textes de Marx) grâce entre autres choses, tantôt à des interventions politiques ${ }^{26}$ et tantôt au progrès scientifique ${ }^{27}$.

N'eût été en somme les circonstances qui ont amené ses successeurs à proclamer le dogme de la détermination " en dernière instance " par l'économie, en déclenchant ainsi des débats spécieux sur l'efficace d'une instance économique abstraitement isolée des autres ${ }^{28}$, la pensée de Marx - malgré l'attention particulièrement insistante qu'elle portait à l'action des facteurs économiques trop ignorés jusque-là - aurait pu être présentée comme une tentative, remarquablement précoce et lucide, pour mettre en relief les interrelations multiples qui composent le tissu social.

24. Marx, K. et Engels, F., L'Idéologie allemande in Pléiade III, p. 1059.

25. Marx, K., Misère de la philosophie in Pléiade I, p. 79.

26. Voir par exemple l'analyse du rôle des législations dans l'établissernent des « enclosures " en Angleterre, dans la huitième section du premier livre du Capital in Pléiade I pp. 1167 et ss.

27. L'Idéologie allemande par exemple fait diverses allusions au rôle de la science dans le développement de l'industrie ; voir, entre autres passages : Pléiade III, p. 1102.

28. Voir à ce sujet le chapitre V sur "l'inutile insistance sur le rapport des instances " du livre cité à la note 14 . 
4) La dernière source de « contradiction » examinée ici est encore plus représentative des débats contemporains. Elle concerne essentiellement l'idée d'autonomie individuelle pour laquelle Marx avait un profond respect mais que semble contredire, sur le plan théorique, la philosophie boliste qu'on associe spontanément à toute perception globale du social et de l'histoire $^{29}$ et, sur le plan pratique, les implications totalitaristes que semble comporter sa philosophie, du moins si l'on en juge par les conséquences de ses applications depuis 1917.

Voyons d'abord ce qu'il en est du problème théorique. Dire, avec Louis Dumont en particulier ${ }^{30}$, que la pensée de Marx est profondément individualiste, c'est dire qu'elle se méfie des explications qui reposent sur une perception des totalités saisies à partir de leur essence même et qu'elle privilégie plutôt comme le feront tant de philosophes positivistes - les explications réductibles, en dernier ressort, à l'action d'entités individuelles. C'est aussi rappeler que l'œuvre théorique de ce penseur se partage entre une critique virulente des spéculations totalisantes d'une certaine "idéologie allemande » et la mise au point d'une théorie économique fondée implicitement sur les postulats individualistes de l'économie ricardienne. Il suffit de se pencher sur la "Critique de la philosophie politique de Hegel ", le seul texte important que Marx ait consacré entièrement à Hegel, pour mesurer combien son auteur se plât à pourfendre les explications holistes où il voit une forme particulièrement pernicieuse de mystification. Pour s'objecter à Hegel dont les vues sur le rôle de l'Idée dans la genèse de l'État lui paraissent trop " mystiques ", il n'hésite pas à soutenir que "l'État provient de la foule telle qu'elle existe, comme la composent les membres de la famille et les membres de la société civile " ${ }^{31}$. Contre cette forme de "mysticisme ", Marx a d'ailleurs poursuivi, tout au long de sa carrière, une lutte impitoyable qui recourait aussi bien à la raillerie qu'à une argumentation " individualiste " apparentée à celle des philosophes analytiques contemporains ${ }^{32}$.

29. Qu'on songe, en particulier, à l'influence exercée par le petit livre de Karl Popper, The Poverty of Historicism, London : Routledge \& Kegan Paul, 1957.

30. C'est l'une des thèses essentielles de l'ouvrage de Louis Dumont cité à la note 6 .

31. "Critique de la philosophie politique de Hegel " in Pléiade III, p. 876 ; voir aussi p. 877.

32. On trouvera une bonne illustration de la chose in $t b \imath d$., pp. 895 à 899 . 
C'est donc cette approche individualiste qui entrerait en contradiction tant avec la mystique de la société communiste à laquelle le nom de Marx est associé qu'avec l'approche dialectique que Marx aurait reprise de Hegel. Il se trouve cependant que d'importantes précisions doivent ici être apportées sur sa double adhésion au communisme et à la dialectique.

On est tellement habitué à associer Marx au communisme qu'on en oublie les critiques qu'il adressait au communisme utopique à la Cabet dont les aspects assez fades ne lui échappaient surtout pas $^{33}$. Or, dans sa préface de 1888 au Manifeste communiste, Engels explique pourquoi Marx et lui ont choisi pour leur option le nom de "communiste " plutôt que celui de "socialiste" : c'est qu'ils estimaient — comme ils l'ont souvent répété - que l'« émancipation des travailleurs doit être l'œuvre des travailleurs eux-mêmes » et que, contrairement au socialisme, le communisme alors existant était vraiment « un mouvement de la classe ouvrière ${ }^{34}$. Il n'est donc pas question là de reconnaître à la communauté une sorte de primauté sur l'individu. Et, là où ils se sont laissés aller à dépeindre cette " société communiste ", Marx et Engels étaient bien loin de sacrifier l'individu à la communauté, comme on le constate dans le plus célèbre des textes consacrés à le faire :

. . personne n'est enfermé dans un cercle exclusif d'activités et chacun peut se former dans n'importe quelle branche de son choix ; c'est la société qui règle la production générale et qui me permet ainsi de faire aujourd'hui telle chose, demain telle autre, de chasser le matin, de pêcher l'après-midi, de m'occuper d'élevage le soir et de m'adonner à la critique après le repas, selon que j'en ai envie, sans jamais devenir chasseur, pêcheur, berger ou critique ${ }^{35}$.

De même, il n'y a pas lieu d'exagérer l'aspect holiste de la dialectique marxienne. Marx a appris de Hegel à penser la totalité sociale et la totalité historique, mais on se rappellera que ces totalités ne peuvent, à ses yeux, être dérivées de l'Idée hégélienne mais qu'elles sont pensées - quoi qu'il en soit du carac-

33. En fait, Marx prend ses distances à l'égard des thèses de la plupart des communistes de son temps, mais il souligne surtout la « platitude » de Cabet. Cf., par exemple, La Sainte Famille in Plérade 111 , p. 572.

34. Engels, F., Préface à l'édition anglaise (1888) du Manifeste communiste in Pléiade I, p. 1487.

35. Marx, K. et Engels, F., L'Idéologie allemande in Pléiade 11I, p. 1065. 
tère un peu rudimentaire de cette gnoséologie - grâce à une « réflexion du mouvement réel, transporté et transposé dans le cerveau de l'homme ${ }^{36}$. En somme, il importe de saisir le " mouvement d'ensemble " de l'histoire et de la société, mais on retomberait dans un mysticisme déplorable si ce mouvement d'ensemble ne pouvait se réduire, en dernier ressort, au résultat des actions, sans doute non-intentionnel, d'individus concrets.

Certes, quand, dans l'analyse économique, il sera question de « capitalistes » ou de «propriétaires fonciers", il ne faudra pas penser à des individus particuliers mais bien plutôt - et c'est pour cela que Marx passe parfois pour anti-individualiste - aux "supports d'intérêts et de rapports de classes déterminés", comme nous en sommes avertis par la préface du Capital ${ }^{37}$. Ce sont donc de tels rapports de classes - et non pas des mobiles psychologiques comme chez les économistes marginalistes qui seront invoqués pour rendre compte des comportements, mais ce n'est pas dire que la réalité sociale, qui manifeste de la sorte ses contraintes propres, doit être pensée comme une totalité irréductible plutôt que comme le résultat historiquement situé des actions nullement coordonnées d'individus concrets sans doute façonnés eux-mêmes par leur propre situation historique.

Reste maintenánt à examiner le volet « pratique » de cette présumée contradiction que l'on a de plus en plus tendance à opposer à Marx à mesure que les diverses expériences politiques inspirées du marxisme semblent déboucher sur des formes diverses de totalitarisme. On a pu, dans un premier temps, condamner ces expériences au nom même du marxisme en alléguant que celui-ci avait été mal appliqué, mais, les déceptions s'accumulant sur ce plan, il devenait tentant de faire remonter à la pensée de Marx elle-même la source du totalitarisme vers lequel convergeaient apparemment ses diverses applications. Or s'il fallait reconnaître dans cette pensée le moindre germe de totalitarisme, on serait en face d'une incohérence majeure de la part d'un si ardent défenseur de l'autonomie individuelle.

Pourtant, ici encore il serait injuste de crier trop vite à la contradiction. Sans doute, Marx ne croyait pas à l'anarchie et

36. Marx, K., Postface de la seconde édition allemande du Capital in Pléiade I, p. 558.

37. Marx, K., Préface de la première édition du Capital in Pléiade I, p. 550 ; le souligné est de Marx. 
son élitisme scientifique contribuait-il à le faire percevoir comme celui qui incarnait, dans le monde socialiste, cette autorité du savoir, jugée plutôt paralysante par ceux qui préféraient se laisser guider par leur instinct. Mais on avouera qu'il en faut plus pour parler de germes de totalitarisme surtout quand on songe que Marx avait, dès 1843 , dénoncé avec une vigueur et une perspicacité probablement inégalées à l'époque les dangers d'une bureaucratie du type de celles qui contribuent tant à infléchir vers le totalitarisme les «applications » de sa pensée ${ }^{38}$.

Aussi est-ce plutôt à la notion de " dictature du prolétariat " que l'on pense quand on croit trouver en elle le germe du totalitarisme. Cette expression apparaît dans une lettre à Weidemeyer datée de 1852 et on la retrouve dans les gloses marginales inspirées à Marx par le programme de Gotha ${ }^{39}$. L'idée elle-même se rencontre ailleurs, en particulier dans le Manifeste communiste et dans l'adresse de 1871 à propos de l'expérience de la Commune ${ }^{40}$. Or, on ne saurait voir, dans cette idée, la moindre justification du totalitarisme qui règne dans les pays d'obédience soviétique, non pas tant parce que la dictature du prolétariat est censée être essentiellement transitoire - on peut toujours alléguer que les transitions sont plus longues que prévu!mais parce que cette dictature était clairement présentée comme celle d'une classe (le prolétariat) sur une autre (la bourgeoisie) ${ }^{41}$. La dictature du prolétariat paraissait nécessaire à Marx parce qu'il était à prévoir que les classes privilégiées n'accepteraient pas de se départir de leurs privilèges autrement que sous la contrainte exercée par un prolétariat qui, on le sait, ne peut compter que sur ses propres moyens. Par exemple, dans le contexte de la Pologne actuelle, il est clair que cette formule (dont le libellé est, admettons-le, assez discutable) justifierait beaucoup plus,

38. Voir la section sur le " pouvoir gouvernemental " dans la "Critique de la philosophie politique de Hegel " in Pléiade III, pp. 915 à 930.

39. Lettre à J. Weydemeyer du 5 mars 1852 in Marx, K. et Engels, F., Lettres sur " le Capital ", Paris : Éditions sociales, 1964, p. 59. "Critique du Programme du parti ouvrier allemand " in Pléiade I, p. 1429.

40. Manifeste communiste in Pléiade 1, pp. 181-183. Marx, K., La guerre civile en France 1871, Paris : Éditions sociales, 1975, pp. 66 à 69.

41. Voir le contexte des deux citations de la nore 39 qui est d'ailleurs conforme à l'idée exprimée par Marx et Engels dans le Manifeste communiste voulant que « le pouvoir politique au sens strict du terme, [soit] le pouvoir organisé d'une classe pour l'oppression d'une autre ", Pléiade I, p. 182. 
aux yeux de Marx, l'action du syndicat Solidarité que celle du gouvernement polonais. Au moment même où il découvrait dans la Commune de Paris ce qui se rapprochait le plus de ce " gouvernement de la classe ouvrière " qu'il espérait voir s'imposer vigoureusement ${ }^{42}$, Marx ne rappelait-il pas à son ami Kugelmann que, dès la rédaction de son 18 Brumaire en 1851-52, il estimait que « la prochaine tentative révolutionnaire en France » ne devrait pas seulement «faire changer de main l'appareil bureaucratico-militaire, mais le briser ${ }^{43}$ ?

Est-ce à dire que les tentatives qui ont abouti jusqu'ici à des régimes totalitaires et "bureaucratico-militaires " ont été des applications incorrectes de la pensée de Marx et qu'il faudrait recommencer à nouveau en espérant faire mieux cette fois ? Il faut certes une bonne dose d'optimisme ou de naïveté pour le conclure, mais ceci n'implique pas forcément la présence d'une sorte de crypto-totalitarisme dans la pensée de Marx. On pourrait en effet expliquer la chose plus radicalement si l'on concluait que ces tentatives malheureuses ne sont en rien des "applications " de la pensée de Marx parce que celle-ci, en définitive, ne serait guère plus "applicable" que celles de Proudhon ou de Thomas More. Marx, après tout, s'est contenté de décortiquer le capitalisme et n'a consacré que bien peu de pages à la construction du socialisme comme tel. Qu'on ait tiré parti du formidable instrument révolutionnaire que constituait le Capital ne fait pas de doute ; mais pourquoi faudrait-il que les monstruosités politiques qu'on a érigées en son nom soient considérées pour autant comme des «applications " de sa pensée, surtout si c'est pour montrer que ces applications entrent en contradiction avec l'expression explicite de cette pensée?

\section{B) Un projet peut-être sans lendemain}

La question qui se pose est donc celle de savoir si Marx est vraiment parvenu à donner des bases « scientifiques » au socialisme. On l'a cru sur la foi de sa conception de l'histoire et de son analyse du capitalisme. Toutefois la société communiste elle-

42. Marx, K., La guerre civile en France 1871, Parıs : Éditions sociales, 1975, p. 67. Le souligné est de Marx.

43. Lettre à Kugelmann du 12 avril 1871 in Marx, K., Marx, J. et Engels, F., Lettres à Kugelmann, Paris : Éditions Sociales, 1971, p. 188. Le souligné est de Marx. 
même, qui est annoncée pour après-demain, reposait sur une conviction bien fragile qui assurait que l'abolition de la propriété privée des moyens de production suffirait à transformer les hommes au point de les rendre aptes à vivre l'idéal socialiste. C'est pourquoi Marx semblait penser que, dans cette société, les hommes pourraient s'adonner à la critique ou à la pêche à la ligne, sans être tentés par d'insatiables besoins ou par un irrésistible goût du pouvoir ${ }^{44}$. C'est pourquoi aussi il ne doutait pas tellement de la possibilité de mettre en place une structure de décision capable de représenter effectivement la classe des travailleurs. Force est de reconnaître que Balkounine, sur ce plan, s'est montré plus perspicace au moment où il prédisait que :

le soi-disant État populaire ne sera rien d'autre que la direction despotique des masses populaires par une nouvelle et nombreuse aristocratie de réels ou de prétendus savants $[\ldots]^{45}$

Quoi qu'il en soit, la « conception matérialiste de l'histoire ", si elle jetait beaucoup de lumière sur le passage de la féodalité au capitalisme, n'éclairait guère celui du capitalisme à un éventuel socialisme. On a cru qu'elle le faisait tant que paraissaient bien déterminés les mécanismes économiques inconscients (e.g. : conflit entre forces productives et rapports de production) qui étaient censés présider à la succession des modes de production. Mais les efforts d'ailleurs remarquables qui ont été faits pour adapter cette théorie au cours effectif de l'histoire ont forcé à en relativiser passablement les fondements et la portée au point de la réduire à n'être plus qu'une expression, certes intelligente et généreuse, de cette foi au progrès social qui a dominé le $\mathrm{XIX}^{\mathrm{e}}$ siècle et que savent encore retrouver les socialistes les plus optimistes de notre époque. Sans nier pour autant la très réelle contribution de Marx à l'intelligence de l'histoire, on ne voit plus très bien quel sens il y a, sur cette base, à faire encore du socialisme une sorte de corollaire d'une théorie prétendue «scientifique $" 46$.

44. Voir à ce sujet le chapitre VII sur « La société de consommation et l'avènement de l'bomo sobrius » du livre cité à la note 14 .

45. Bakounine, L'État et l'anarchie, cité sans références par Lefebvre, H., op. cit., p. 260.

46. Voir à ce sujet le chapitre VI sur " Ambivalence et pertinence de la philosophie marxiste de l'histoire » du livre cité à la note 14 ; ce texte est paru également dans les Actes du 
Par ailleurs, l'analyse économique de Marx, nonobstant ses remarquables qualités, n'a pas manqué de se heurter, au $\mathrm{XX}^{\mathrm{e}}$ siècle, à de sérieuses difficultés qui en ont considérablement réduit la portée. D'une part, la loi de la baisse tendancielle du taux de profit, qui garantissait à long terme l'épuisement des ressources du capitalisme au bénéfice d'un autre mode de production, s'est révélée plutôt équivoque et finalement assez peu significative. D'autre part, la théorie de la valeur-travail, la base de la théorie de la plus-value, s'est montrée incapable de rendre compte des prix et, après avoir suscité, pendant cent ans, les plus ingénieuses tentatives de réinterprétation, parvient tout au plus à stimuler la vigilance critique de ceux qui continuent à dénoncer, au nom de Marx, l'exploitation capitaliste ${ }^{47}$.

Bref, les deux « grandes découvertes » qui, aux yeux d'Engels, permettaient de parler de socialisme scientifique se sont vues réduites à des considérations de portée beaucoup plus limitée qu'on ne croyait d'abord. Dans le cadre de ces limites, que, de façon tout à fait normale, on a cru longtemps pouvoir dépasser, la pensée de Marx n'a certes pas manqué de cohérence ni de perspicacité. Ces qualités cependant n'auraient pas suffi à en faire la pensée à part les autres qu'elle est devenue. Un statut aussi exceptionnel ne se justifiait que parce qu'on y a vu cet événement théorique que constituait la jonction du socialisme et de la science ou, si l'on préfere, la jonction des aspirations à la justice et à la vérité. Or, c'est cette " jonction ", qui aurait sans doute constitué une conquête grandiose et peut-être démesurée, qui, après cent ans, se révèle si précaire qu'on est en droit de se demander si elle a vraiment eu lieu.

\section{CENT ANS DE MARXISME}

Reste donc, en terminant, à examiner, bien sommairement à vrai dire, la façon dont l'événement théorique qui donnait toute sa signification à la pensée de Marx a été vécu à diverses époques de l'histoire du marxisme.

colloque La philosophie de l'bistoire et la pratıque historienne d'aujourd'bui, paru sous ce titre aux éditions de l'Université d'Ottawa en 1982, cf : pp. 293-308.

47. Voir à ce sujet les deux dernières sections du chapitre III du livre cité à la note 14 ; j'expose de façon plus systématique ces idées sur la théorie de la valeur dans un texte intitulé « Le sprincipe de conservation de la valeur et le problème de la transformation » qui doit paraître chez Maspero dans un ouvrage collectif consacré à la théorie de la valeur. 
Au lendemain de la mort de Marx, le marxisme fut d'abord dominé par la personnalité d'Engels qui s'est d'ailleurs chargé de compléter le versant scientifique (matérialisme historique) auquel se réduisaient, à toutes fins pratiques, les œuvres alors publiées de Marx - par un ambitieux versant philosophique (matérialisme dialectique). Ce marxisme, n'ayant pas encore connu d'applications politiques, ne se trouvait donc - quel que soit le sens qu'il faut donner à ces termes - ni "vérifié ", ni " contredit» par les faits. Par contre, il était en général fort respecté de ceux qui ne feignaient pas de l'ignorer ou de le mépriser parce que, comme on l'a vu, il pouvait passer pour la seule entreprise d'explication scientifique des phénomènes sociaux qui, du fait de l'attention qu'elle portait aux conditions matérielles de leur production, correspondait assez bien à l'idée qu'on se faisait alors de la science. Pour les marxistes de cette époque, l'adhésion au marxisme pouvait donc se justifier assez aisément, vu l'importance manifeste de l'événement théorique qu'Engels avait déjà caractérisé comme l'avènement du socialisme scientifique.

Cette vision assez dogmatique et orthodoxe du marxisme s'est plus ou moins perpétuée jusqu'à nos jours au sein des partis marxistes qui, en 1917, prenaient avec Lénine une nouvelle orientation politique ; mais une série de données nouvelles allaient bientôt précipiter la naissance de perceptions nouvelles de l'événement théorique que représentait la pensée de Marx.

Sur le plan théorique, les thèses économiques et historiques de Marx avaient commencé à se heurter à d'importantes difficultés comme celles évoquées plus haut. Par ailleurs, les économistes marginalistes, de plus en plus satisfaits de leurs théories qui se déployaient déjà en systèmes formalisés, se sentaient plus rassurés face au marxisme; parallèlement, une sociologie "bourgeoise ", inspirée par Durkheim, Weber et Pareto en particulier, commençait à contester avec succès un terrain que le marxisme, jusque-là, avait eu l'air d'occuper seul.

Sur le plan politique toutefois, les choses changeaient brusquement en sa faveur : la crise des années 30, en Occident, semblait donner raison à Marx puisque, selon toute apparence, le capitalisme s'apprêtait à rendre l'âme, alors même qu'en URSS 
les succès de la planification et de la croissance accélérée étaient impressionnants. Cette fois, c'était moins les qualités internes de l'analyse que les succès de ce qui se donnait comme une prévision fondamentale de la théorie marxienne qui soutenait l'idée que celle-ci, malgré des difficultés passagères qu'on pouvait lui opposer, correspondait à rien de moins qu'à l'avènement du socialisme scientifique.

Une telle interprétation des choses satisfaisait surtout ceux pour qui le marxisme était avant tout une analyse économique. Pour d'autres, plus attentifs aux questions philosophiques, c'était surtout le ton de plus en plus dogmatique que prenait la version officielle d'un matérialisme dialectique mis au service du parti et du pays de la révolution qui faisait surtout scandale. Fort heureusement, vers cette époque, la publication des écrits de jeunesse de Marx, où s'exprimait explicitement une philosophie plus humaniste et moins dogmatique que le matérialisme dialectique, allait donner un élan nouveau au " marxisme occidental ». L'adhésion au marxisme continuait d'y être une adhésion au socialisme scientifique, mais ce socialisme s'enrichissait d'une philosophie qui donnait un sens à l'histoire et aux valeurs humaines.

Malgré les inquiétudes que commençaient à susciter le redressement inattendu du capitalisme et l'orientation nouvelle de la Russie stalinienne d'où filtraient déjà des rumeurs sur les procès de Moscou, ce marxisme humaniste connut un second souffle du fait même de la guerre et surtout de la résistance où l'héroïsme de tant de marxistes militants suffisait presque à convaincre les autres que l'histoire ne pouvait que leur donner raison et favoriser l'avènement de la société humaniste et antifasciste auquel ils travaillaient si intensément.

Les choses se gâtèrent au lendemain de la guerre, et surtout au cours des années 50 , avec la pénible prise de conscience du caractère totalitariste et impérialiste de l'expérience soviétique. La situation était d'autant plus délicate pour les marxistes que l'Occident s'engageait dans une ère de prospérité exceptionnelle et que les dangers du développement technologique auxquels leur esprit critique les rendait justement sensibles réduisait la pertinence des thèses marxistes traditionnelles qui avaient ten- 
dance à valoriser systématiquement la science et la technologie. Qui plus est, le matérialisme lui-même avait cessé d'être la philosophie privilégiée de la science, car l'épistémologie moderne, fort suspecte à l'égard de l'empirisme, se faisait nettement plus subtile que celle d'Engels.

C'est cette situation embarrassante qui fut brusquement oubliée avec le marxisme des années 60. Ceux que l'évolution de l'Union soviétique inquiétait pouvaient maintenant se tourner vers l'expérience plus enthousiasmante et plus prometteuse d'une Chine en pleine révolution culturelle. Au même moment, Althusser allait tirer parti de l'humeur anti-empiriste de l'épistémologie pour proposer une lecture toute nouvelle du marxisme : grâce à celle-ci, la "science marxiste " n'aurait plus à s'embarrasser de vérifications empiriques, sans devoir se tourner pour autant vers une philosophie humaniste de moins en moins adaptée aux questions soulevées par un monde dominé par la pensée scientifique ; cette "science marxiste », désormais, allait être éclairée et protégée de l'idéologie par une épistémologie inédite qu'Althusser se chargeait de "lire » dans le Capital. Pendant quelques années, on fut manifestement enthousiasmé par des perspectives de ce genre ; on pouvait à nouveau se convaincre que la critique sociale et la lutte révolutionnaire étaient cautionnées par une science d'autant plus étrangère aux critères de la science traditionnelle qu'elle reléguait au rang d'idéologie les théories qui la contestaient et qu'elle se donnait pour un " paradigme "scientifique radicalement nouveau ${ }^{48}$.

C'est cette construction théorique un peu fragile qui semble s'être brusquement défaite au moment même où s'estompait l'espoir suscité par le socialisme à la chinoise et où se multipliaient, partout en Occident, les manifestations d'une méfiance croissante à l'endroit de toutes formes d'intervention de l'État. Et ce sont ces circonstances réunies - et quelques autres encore - qui ont peu à peu dénoué les liens qui semblaient relier solidement le socialisme et la science, en plongeant ainsi le marxisme dans cette période creuse qui devait être celle d'un centenaire célébré, somme toute, avec assez peu d'éclat.

48. Pour l'analyse des traits propres à ce marxisme des années soixante, voir en particulier l'introduction et la conclusion de l'ouvrage cité à la note 14. 
Est-ce à dire que, cette fois, Marx est bel et bien mort ? On l'a pensé chaque fois que s'effritaient ainsi les espoirs trop. bruyants d'une période antérieure. Ce serait donc assez hasardeux de le soutenir aujourd'hui, d'autant que ce "marxisme " qui semble périodiquement retrouver une vigueur nouvelle ne s'embarrasse guère d'une fidélité excessive à l'égard de Marx. En fait, ce qui fut chaque fois réactivé sous ce nom, c'est moins la pensée d'un des plus grands penseurs du XIX ${ }^{\mathrm{e}}$ siècle que l'événement théorique que cette pensée $a$, pour la première fois, incarné pleinement. Or, comme il est douteux que l'humanité puisse jamais se résoudre à abandonner définitivement le rêve de faire coïncider enfin ses aspirations à la justice sociale et à la vérité scientifique, il est permis de penser que ce " marxisme ", sous quelque forme que ce soit et au risque de trahir encore d'autres aspects de la pensée de Marx, soit, en quelque sorte, perpétuellement condamné à renaître de ses cendres.

Département de philosophie Université de Montréal 